Derechos reservados de El Colegio de Sonora, ISSN 0188-7408

\title{
Conocimiento y estudios sobre el agua subterránea en la Costa de Hermosillo
}

José Luis Moreno Vázquez*

Resumen: En este trabajo se analiza el papel que desempeñó la evolución del conocimiento científico en materia de agua subterránea en el desarrollo de una región agrícola del noroeste del país. En la introducción, se aborda el contexto económico y la visión dominante que existía sobre la disponibilidad y aprovechamiento del agua que hicieron posible el nacimiento de la Costa de Hermosillo a mediados de los años 40. En el primer apartado, se describe el conocimiento que había sobre el agua del subsuelo en ciertas zonas del país y del estado de Sonora antes de dicho nacimiento. En el segundo, se muestran las diferentes estimaciones realizadas acerca del potencial de agua subterránea a nivel nacional y estatal, así como los métodos empleados para tal efecto. En el tercer apartado, se discuten las diversas estimaciones efectuadas sobre la recarga natural del acuífero de la Costa a lo largo de la década de los años 60. Por último, en el cuarto se ofrece un resumen de las conclusiones derivadas de este análisis y los riesgos de la falta de conocimiento actualizado sobre este acuífero ante la posible puesta en práctica de nuevos proyectos de aprovechamiento de agua.

\footnotetext{
* Investigador de El Colegio de Sonora, se le puede enviar correspondencia a Obregón 54, Centro, C.P. 83000. Tel.: (62) 1265 51, fax: (62) 1250 21. Correo electrónico:jmoreno@ colson.edu.mx
} 
Abstract: This work analyzes the role played by the evolution of scientific knowledge with regard to groundwater in the development of an agricultural region in the northwest of the country. The introduction presents the economic context and the prevailing view of the availability and exploitation of water which made possible the origin of the Costa de Hermosillo in the mid-40s. The first section describes the knowledge of groundwater in certain areas of the country and in the state of Sonora that existed before the origin of the Costa.The second section shows different estimates of groundwater potential at both national and state levels, as well as the methods used for these estimates The third section dis cusses various estimates of the natural recharge of the Costa aquifer throughout the sixties. Finally, the fourth section gives a summary of the conclusions derived from this analysis, and the risks stemming from the lack of up-to-date knowledge of this aquifer prior to possible implementation of new projects for the utilization of water.

\section{Introducción}

Este trabajo tiene como objetivo mostrar el contexto de conocimiento hidrológico en el que se dio el nacimiento de la región agrícola de la Costa de Hermosillo a fines de la década de los años 40, así como la forma en que evolucionó dicho saber en cuanto al manto acuífero que la alimenta en el periodo del auge productivo de los años 50 y 60. La hipótesis central es que el conocimiento de las ca racterísticas principales de los recursos hidrológicos del subsuelo fue a la zaga de su explotación en gran escala. Tal desfase provocó que la extracción del líquido se realizara sin tener certeza de su potencial real de aprovechamiento. Entre los impactos negativos estuvieron el abatimiento del acuífero y la presencia de indicios de intrusión sa- 
lina. No obstante, cuando dicho conocimiento se adquirió a fines de los años 60, este saber no se utilizó para llevar a cabo una explotación adecuada del recurso, sino para extraer mayores cantidades de agua del subsuelo, lo que causó la agudización de los impactos antes señalados.

Entre los elementos de contexto más relevantes para entender el desarrollo agrícola de la Costa y la explotación de su acuífero inicia dos en 1945, se encuentra el discurso oficial centrado en la necesidad de expandir la economía del país y en "ganar la batalla por la producción", planteado por el presidente Manuel Ávila Camacho en el marco de la Segunda Guerra Mundial. La estrategia consistía en continuar con la apertura de nuevas tierras al cultivo e intensificar las políticas de irrigación y colonización promovidas por los gobiernos posrevolucionarios, en particular, en las áreas "inhóspitas, vacías, baldías u ociosas" del norte de México. Esta estrategia incluía como novedad la asignación de un nuevo papel a la agricultura: apoyar el proceso de industrialización del país y satisfacer las necesida des de una población futura que se estimaba sería mucho mayor en número a la que se tenía en aquel entonces.

Dicha estrategia continuaría, con pequeñas diferencias, en las administraciones posteriores de los presidentes Miguel Alemán y Adolfo Ruiz Cortines, dando como resultado una superficie total puesta bajo riego de aproximadamente 2 millones de hectáreas (entre nuevas y mejoradas) en el periodo 1940-1958. Otro resultado importante fue el cambio cualitaivo que se dio en la superficie de $\mathbf{1 . 7}$ millones de hectáreas (ha) de particulares. De un $\mathbf{8 \%}$ del total de la superficie bajo "riego seguro" con pozos, se pasó en el mismo lapso a 41\%, lo cual representó un aumento de 150 a 700 mil hectáreas bajo este tipo de riego, así como la disminución del uso del riego eventual (Orive Alba, 1960:176-178).

Otro de los elementos de contexto fue la visión dominante que se tenía sobre los recursos hidrológicos del país. Si bien se reconocía la carencia de esta riqueza natural en la mayor parte del territorio na cional ( ya que $93 \%$ se consideraba árido o semiárido), se proponía que ésta podía ser solventada gracias a la innovación tecnológica, los avances de la ingeniería hidráulica, la disponibilidad de recursos financieros y el paso del tiempo. Considerada "hija de la segunda re- 
volución industrial", esta innovación tecnológica implicó el uso de nuevos materiales como el concreto, nuevos diseños de presas, el empleo del ferrocarril y motores de combustión interna, así como el desarrollo paralelo de la energía eléctrica (Aboites, 1999:159).

La confianza en el papel de la ingeniería hidráulica para impulsar el desarrollo agrícola en gran escala se reflejó en la participación que tuvieron algunos de sus exponentes principales en la creación y dirección de instituciones como la Comisión Nacional de Irrigación (CNI) en 1926 y la Secretaría de Recursos Hidráulicos (SRH) en 1946, entre los que destacaron Javier Sánchez Mejorada y Adolfo Orive Alba. En ese año, este último funcionario señalaba que en las zonas áridas había recursos hidráulicos posibles "para regar 5 millones de hectáreas" (Orive Alba, 1946:103). Era tal su optimismo y su confianza, que decía que si se continuaba con el mismo "ritmo de tra bajo" y el presupuesto asignado en tal año, entonces todo era una simple cuestión de tiempo:

...en unos 27 años se habrían ejecutado todas las obras de riego necesarias para aprovechar todos nuestros recursos hidráulicos en las zonas áridas y semiáridas de México y en otros 13 años habríamos ejecutado las obras necesarias para regar los dos millones de hectáreas en las zonas semihúmedas, si ello resulta necesario, como creemos. Ahora bien, un lapso total de 40 años - que aun se acortańa si los presupuestos asignados a irrigación siguen la progresión creciente que hasta la fecha- sería muy corto en la vida de un país para lograr obtener el total aprovechamiento en riego de sus recursos hidráulicos (Orive Alba, 1946: 126).

Al final de su texto, el mismo funcionario señalaba la oportunidad que se le presentaba al pás y a los estados del norte en relación con otras naciones del planeta que sufrían las consecuencias de la conflagración mundial:

...en estos momentos en que $\mathrm{e}$ mundo aún se debate en una guerra sangrienta provocada por páses que quisieron conquistar nuevos territorios por la fuerza de las armas, podemos estar or- 
gullosos, nosotros, los mexicanos, de que nuestro país también esté conquistando nuevos territorios, pero paćfica, civilizadamente, mediante su política de irrigación, mediante la conquis ta de sus propios desiertos (I bídem).

Esta visión se centraba básicamente en el conocimiento que se tenía del agua superficial. En cuanto al agua subterránea, todavía era escaso lo que sabía sobre ella, aunque había muchos sitios dispersos en el territorio nacional en donde se explotaba desde hacía varios años, como se expone en el siguiente apartado. Debido a este desconocimiento de los acuíferos, dentro de la recién creada SRH se estableció en 1947 la Dirección de Geología, cuyo primer jefe fue el ingeniero geólogo y geofísico Alfonso De la 0 Carreño. Entre los objetivos principales de esta nueva dependencia estaba "hacer el estudio sistemático y el inventario de los recursos hidráulicos del subsuelo". Para tal efecto, "se deberían emplear los sistemas más modernos, como los métodos geofísicos de exploración o nuevos sistemas usando ondas de radar desarrolladas en estos últimos años con motivo de los adelantos logrados en la pasada guerra mundial" (Orive Alba, 1960:14).

\section{Las primeras extracciones y exploraciones en México y Sonora}

Como se mencionó antes, al terminar la década de los años 40 , el conocimiento que se tenía de la cantidad de agua subterránea existente en el subsuelo del país era todavía escaso. No obstante, según Paul Waitz, reconocido especialista del tema en aquella época, este conocimiento impreciso y la carencia de estudios sobre sus posibilidades reales de aprovechamiento no habían sido obstáculos para su explotación con éxito en muchas zonas de la república en la década de los años 30. Tal explotación se hacía para abastecer del líquido a las ciudades de Guadalajara y Monterrey, o con diversas finalidades en las inmediaciones de las ciudades de México, Celaya y León. También se extraía líquido en varias haciendas del centro del país, en re- 
giones agrícolas como La Laguna, en estaciones de ferrocarril como Pesqueira al norte de Hermosillo, Sonora, y en áreas fronterizas como el valle de Juárez (Arreguín, 1998: 35-37).

De acuerdo con Waitz, en esos años no se habían hecho capta ciones de agua subterránea en gran escala y mucho menos en forma sistemática por parte del Estado, ya que los más connotados aprovechamientos eran realizados siempre a cuenta y riesgo de empresas particulares. El estudio de los aprovechamientos - decía- "no ha bía pasado más allá de reconocimientos ligeros, como en el valle Trincheras, en el distrito de Altar, Sonora".1 De hecho, algunos de los trabajos realizados en zonas semiáridas de Sonora fueron publicados a mediados de los años 20, como los de los ingenieros geólogos Maximino Alcalá (1927) y Luis Blásquez (1926). El primero mencionó la existencia de un manto acuífero situado a 180 metros de profundidad, producto de las perforaciones hechas con anterioridad por Ferrocarriles en Agua Zarca y Casita en el río Magdalena cerca de Nogales; en tanto el segundo mencionó la existencia de "abundantes aguas freáticas" en las cuencas de los níos Concepción y Sonoyta.

Entre los primeros estudios realizados para el aforo de aguas subterráneas,Arreguín (1998: 36) resaltó un trabajo del ingeniero José S. de la Vega publicado en 1933 sobre el río Pilón en Nuevo León, que utilizó el procedimiento eléctrico y que era considerado por su autor como "el más preciso y expedito de todos los existentes". Este método se aplicaba por primera vez en nuestro país y demostraba el interés por cuantificar el recurso, aunque se tratara solamente de aguas freáticas. ${ }^{2}$ También resaltó un trabajo del ingeniero Luis Echeagaray, quien en 1938 publicó un trabajo sobre los

\footnotetext{
1 Los trabajos principales de Paul Waitz fueron publicados en la revista Irrigación en M éxico entre 1930 y 1943.

2 El procedimiento eléctrico consistía en la instalación de dos pozos someros ubicados a cierta distancia y en dirección de la corriente subterránea; el de aguas arriba se contamina con sal, y con un amperímetro, o bien aparatos que miden la conductividad eléctrica, se registra el momento en que llega la contaminación al pozo ubicado aguas abajo, registrando desde luego el tiempo de llegada para aś estimar la velocidad de filtración, que relaciona la distancia entre pozos y el tiempo de recorrido.
} 
tres métodos existentes para aforar aguas subterráneas: a) el ya mencionado procedimiento eléctrico, b) los procedimientos de clorantes, y c) el procedimiento basado en la ley de Darcy, conocida desde 1856 (Arreguín, 1998: 39).

Un estudio sobresaliente fue el del ingeniero geólogo Gonzalo Vivar, llevado acabo en 1934 en una parte de la región que años después sería el distrito de riego por bombeo de Santo Domingo, Baja California Sur, la cual mostraría cierta similitud de características naturales con la región de la Costa de Hermosillo. En dicho estudio se incluyó una estimación gruesa del volumen de agua subterránea aprovechable y recomendaba, por prudencia y antes de emprender cualquier obra costosa, realizar un bombeo experimental, así como efectuar una investigación del abatimiento del nivel hidrostático y el posible agotamiento o invasión de agua salada "para juzgar acerca de la cantidad de hectáreas irrigables". Esto llamaría la atención deArreguín (1998: 38), pues por esas fechas no se tenían experiencias en México de intrusión salina del mar, siendo tal vez ese señalamiento producto de "referencias bibliográficas".

Para dicho autor, estos fueron los años que conformaron la primera etapa de la historia de la geohidrología en México, que él denominó "los albores de la geohidrología" y que comprendió de 1890 a 1935. Sus características principales fueron: la relativa abundancia de agua ante una población reducida; la resolución de problemas geohidrológicos que no requerían de estudios o investigaciones profundas para aprovechar el agua subterránea; los limitados estudios geohidrológicos que brillaban en un medio caracterizado por "burdos lirismos y prácticas de zahoríes" y los métodos carentes de todo principio científico, o bien, en el mejor de los casos, en los escasos conocimientos y dudosa experiencia de perforadores Las obras más espectaculares y de mayor mérito fueron las grandes obras hidráulicas de captación y conducción como el acueducto de Xochimilco, que captaba el líquido de manantiales cercanos para posteriormente enviarlo a la ciudad de México (Arreguín 1998: 40).

De hecho, no importaban mayormente diversos aspectos de la geohidrología porque simplemente no se necesitaban.Así, esta eta pa se distinguió por: 
...concentrarse en la simple búsqueda del agua subterránea y, desde luego, su incipiente aprovechamiento ante necesidades y requerimientos modestos, casi siempre satiffechos por fuentes locales cuya potencialidad bastaba y hasta sobraba, mediante ma nantiales, norias someras, galerías filtrantes y pozos de poca profundidad (Arreguín 1998: 25).

Otro trabajo sobresaliente realizado en esta etapa en la región de estudio fue el efectuado por el ingeniero en minas Teodoro Flores en una porción de la cuenca del río Sonora. Entre sus objetivos estaba la realización de un reconocimiento geológico en la parte central de esta región, en donde se proyectaba construir una presa para la irrigación de las tierras del valle de Ures, $100 \mathrm{~km}$ al noreste de la capital del estado. Sobresale, por su mención a la escasez de agua y a la poca probabilidad de encontrar agua artesiana en terrenos de lo que sería después al distrito de riego por bombeo de la Costa de Hermosillo:

El agua en esta región es escasa y últimamente se ha buscado perforando pozos bastante profundos (SantaTeresa, Tejunco, San Miguel, Pimienta y otros), algunos de los cuales han alcanzado las aguas freáticas a los 60 metros de profundidad. En la época de nuestra visita a esta región (1922) el pozo de SantaTeresa había llegado a una profundidad de 128 metros sin haber encontrado, hasta entonces, agua brotante ni ningún macizo rocalloso, sino solamente arenas o materiales fragmentarios, pudiendo así apreciarse por esta perforación el espesor que tienen hacia la costa los depósitos de acarreo, el que parece ser muy considerable (Flores, 1929:18).

Más adelante reafirmaba su opinión sobre las aguas artesianas de la manera siguiente:

Es poco probable, en nuestro concepto, que se encuentren aguas de esta naturaleza en ese sitio porque hasta ahora, no obstante lo profundo de esas perforaciones, se han encontrado sola mente terrenos arenosos sueltos o detríticos sin haber cortado 
ninguna formación sedimentaria en la que pudiera existir alguna capa impermeable que tuviera captada alguna agua a presión; en cambio sí es posible que las aguas sean ascendentes en esos pozos, sin ser brotantes y que se pueda proveer de agua suficiente a esas feraces tierras del ex-Distrito de Hermosillo bombeando el agua (Flores, 1929:25).

Un aspecto importante del estudio de Flores fue que incluyó las observaciones del geólogo E.T. Dumble cuando trabajaba al servicio del Ferrocarril Sud-Pacífico a principios de siglo. En forma contraria a lo expuesto por aquél, éste hace referencia a la presencia de aguas subterráneas en las tierras de los valles de Ures, Hermosillo y San José de Guaymas. Llamando la atención sobre el porvenir agrícola de algunas regiones de Sonora y en especial la "feracidad" de esas tierras, apunta que dichas aguas podrían utilizarse para su irrigación perforando "pozos más o menos profundos". Esta opinión la sus tenta en los resultados obtenidos al perforar un pozo en la estación Maytorena del ferrocarril en el valle de Guaymas y "cortar" una importante capa de agua de $\mathbf{4 6} \mathrm{m}$ de espesor. Además de sus hallazgos, es importante destacar su premonición de lo que acontecería años después en los valles costeros de Sonora y, en particular, en la Costa de Hermosillo:

Por el resultado tan satisfactorio como el que queda dicho es de esperar y desear que otros $s$ animen a hacer perforar pozos en éste y otros valles similares. Juzgando por los estudios geológicos que he podido practicar, éste es el único pozo que ha alcanzado un volumen de agua semejante (...) La configuración y declive que presenta el terreno me hace creer también que en algunas partes del valle podrían descubrirse pozos artesianos o pozos comunes, mas en la mayoría de los casos el agua tendría que extraerse por medio de bomba. Para las necesidades más precisas y la explotación agrícola en pequeña escala la extracción puede hacerse por medio de molinos de viento o máquinas de gasolina, mas al tratarse de grandes acopios para el riego será indispensable el uso de potentes bombas de vapor para las que se vislumbra la probabilidad de que en día no lejano habrá abundancia de 
combustible barato. (...) Aun cuando para llegar a conclusiones definitivas tocante a la cantidad de agua que pueda encontrarse, será preciso perforar algunos otros pozos en parte más lejana, en el centro del valle, hay aparentemente líquido suficiente pa ra el regadío de huertas, viñedos y vergeles y tal vez con la ayuda del agua fluvial levantar abundantes cosechas de cereales y forra jes. Posible es que con perforaciones más profundas se obtuviesen resultados más satisfactorios todavía (Flores, 1929:85-86). 3

Al final, Dumble pone como ejemplo de lo que podía realizarse con abundante provisión de agua a la "floreciente agricultura" del vecino estado de California y las "múltiples industrias" que de ella se derivan, rematando su argumentación con el siguiente comenta rio:

Es innegable que el extenso valle de Guaymas, aunque apa rentemente estéril e improductivo, es rico en suelo y agua. Depende, por tanto, de la energía de los ciudadanos de Sonora el convertirlo en uno de los más bellos jardines de esta progresista y atractiva entidad (Dumble, 1901).

Flores, por su parte, (1929:86), no se quedaría atrás al reafirmar la "urgente necesidad" de ejecutar las obras de la importancia de la presa y otras de igual o mayor magnitud para regar lastierras de U res y Hermosillo, así como:

...las situadas hacia la costa que son probablemente las más fértiles, obras que convertirían propiedades que son ahora eria zas e improductivas y prácticamente sin valor, en fuentes de producción para sus dueños, lo que en definitiva redundará en provecho de la riqueza nacional.

Años más adelante, en la década de los 50, Teodoro Flores ocupó el cargo de director del Instituto de Geología de la unAM. Fue en

3 El trabajo citado era: "El valle de Guaymas", E. T. Dumble. 
su periodo cuando el citado De la 0 Carreño publicó sus primeros trabajos sobre las provincias geohidrológicas de México en el boletín de dicha institución y que son comentados en el siguiente apartado.

Cabe resaltar que varias décadas antes de la conformación del distrito de riego de la Costa de Hermosillo, el antropólogo estadounidense William McGee en su obra sobre los indios seris elaborada a fines del siglo XIX, mencionó que algunos ranchos de la región como el Costa Rica se abastecían de las filtraciones subterráneas del río Sonora, en tanto otros ranchos como el Libertad y SantaAna, situa dos más hacia el norte, se abastecían de las filtraciones del río $\mathrm{Ba}$ coachi (McGee, 1980: 47 y 28).

En ese entonces, la riqueza acuífera existente en el subsuelo del Bacoachi era ya mencionada, si bien todavía no era cuantificada. De acuerdo con dicho autor, el Bacoachi era una típica corriente fluvial de la provincia sonorense. Según la estación, mostraba tramos pequeños de 8 a 24 kilómetros en donde corría por la superficie de arenas, y tramos largos; allí el río se introducía al subsuelo convirtiéndose en "aluvión arenoso por 80 kilómetros", distancia en la cual podían hallarse "pozos cada vez más profundos". Después, pa saba a la "fase delta" y penetraba en el noreste del territorio seri, en una zona caracterizada por bosques de mezquites excepcionalmente robustos. Este río - que él consideraba nacía $320 \mathrm{~km}$ al noreste de Bahía Kino- durante y después de las tormentas se transforma ba en "un río que llega a tener el caudal del Ohio o el Rin" y recorría tumultuosamente $\mathbf{2 4 0}$ kilómetros para finalmente hundirse en las arenas del Desierto Encinas, a 50 u 80 km de la costa. Además - continuó- desde su nacimiento "suficiente cantidad de agua atraviesa las barreras, para abastecer a los pozos profundos del aba nico terminal", aś como a dos de los ranchos antes mencionados (McGee, 1980: 45-47).

También es de resaltar que en el grupo de trabajo que llevó a ca bo los estudios en la zona seri se encontraba Willard D. Johnson, quien fue el fotógrafo y topógrafo de la segunda expedición efectuada en 1895 y contaba con la autorización de Investigaciones Geológicas de los Estados Unidos. 


\section{Las primeras estimaciones sobre el agua del subsuelo}

De acuerdo con el titular de la SRH, en 1947 las estimaciones más confiables acerca del agua era que del $100 \%$ de la precipitación total anual de México, 17\% $s$ infiltraba al suelo para formar depósitos y corrientes subterráneas, $60 \%$ se evaporaba y transpiraba, y $23 \%$ escurría superficialmente. Esto significaba alrededor de $263 \mathrm{mil}$ millones de metros cúbicos de agua en el subsuelo al año "cuyo aprovechamiento para riego, abastecimiento de agua potable 0 usos industriales es de vital importancia para el país" (OriveAlba, 1960: 14-16).

Otras estimaciones realizadas posteriormente por el citado Alfonso De la 0 Carreño (1954) y el ingeniero Luis Echeagaray (1958) calcularon un volumen de $\mathbf{2 7 0}$ mil y 152 mil millones de metros cúbicos, respectivamente. La segunda estimación señaló gros so modo y en forma preliminar que se "podría llegar a aprovechar" $\mathrm{d}$ $42 \%$ del volumen total de agua en el subsuelo, es decir, $64 \mathrm{mil} \mathrm{mi-}$ llones de metros cúbicos. 4

Ese mismo año de 1958, el ingeniero civil Jorge Tamayo, con amplia trayectoria en la administración pública y la academia, ofreció sus propias estimaciones. Consideró "excesiva" la cifra de 211 mil millones de metros cúbicos que calculó otro ingeniero, Andrés García Quintero. Propuso - basado en datos de De la 0 sobre valorización de las superficies llanas del país- un volumen total de 185 mil millones de metros cúbicos y una cifra de 27 mil millones de metros cúbicos como volumen anual que se infiltra a los depósitos "que puede extraerse sin afectar los almacenamientos subterráneos". Esto es, $58 \%$ menos del volumen de agua calculado por García Quintero. En la determinación de este valor menor de agua influyó un fenómeno que él ya había observado en los acuíferos.

\footnotetext{
4 La diferencia entre la estimación de Echeagaray y las dos primeras se derivaba de que en aquélla se utilizó el método deThornthwaite, que si bien dio buenos resultados para calcular las demandas de riego, no lo fue así para tratar de obtener evapotranspiración real. Por su parte, el método empleado por De la 0 Carreño y Orive fue la ecua ción de E. Martonne, procedimiento también criticado porque la evapotranspiración se determinaba mediante fórmulas empíricas de dudosa certeza, ya que al ser su rango de error muy amplio, podía caber en ella varias veces el valor real de la infiltración.
} 
Tal cifra parece excesiva y en cierto modo se contradice con la realidad que nos está demostrando en las zonas de explotación que la infiltración tiene valores menores, razón por lo que tan luego se hace un bombeo intensivo, descienden los niveles ( $\mathrm{Ta}$ mayo: 1958: 92).

Del total calculado porTamayo, el $14 \%$ del volumen acuífero, es to es, 4 mil millones de metros cúbicos, se localizaban en la planicie costera de Sonora y en la parte norte del estado de Sinaloa hasta el río del mismo nombre Las regiones con mayor potencial en el país eran: el resto de la planicie costera del Pacífico hasta el río Verde en Oaxaca ( con $31 \%$ del volumen total) y las vertientes interiores de la Altiplanicie Mexicana (con 29\%).

En su estudio publicado en dos partes, el citado De la 0 Carreño da cuenta del avance que se tenía en esta materia y del reto que se tenía enfrente. Apunta que la explotación de la riqueza acuífera del subsuelo había empezado de manera general "hasta hace apenas unos tres años", es decir, en 1948. Llama la atención sobre la necesidad de incrementar el área de riego en el país, no sólo con aguas superficiales sino también con las subterráneas, debido a que, según cifras de Orive Alba, ya titular de la SRH, 14 millones de hectáreas susceptibles de cultivo en el país no podían regarse por falta de agua, 7 millones contaban con agua superficial y 2 millones no necesita ban riego por encontrarse en zonas húmedas. Todo esto justificaba la necesidad de que "conozcamos nuestros recursos naturales geohidrológicos". A raíz de la creación de la citada Dirección de Geología en 1947 y años antes, en 1939, de la Oficina de Geohidrología en la CNI - decía- se "despertó en gran escala el interés público por aprovechar nuestros recursos de aguas subterráneas", lo cual incluía la apertura de perforaciones hechas por la propia secretaría y la cooperación de otras instituciones o particulares (De la 0 Carreño, 1951: XI-XIII; 1954: 52-53).

En este trabajo se calculó el escurrimiento superficial, la evaporación y la infiltración en las provincias geohidrológicas de Sonora, entre las que estuvieron las denominadas Seri, Bacoachi y Hermosillo. En las tres provincias, el porcentaje de agua infiltrada, con respecto al total de precipitación, osciló entre 31 y 42\%. La infiltración 
global calculada fue de 74, 718 y 3,143 millones de metros cúbi$\cos (\mathrm{Mm} 3)$ de agua al año, respectivamente. Este volumen no implicaba el potencial disponible para su extracción del subsuelo en cada una de las provincias, sino sólo una estimación del líquido que se infiltraba de acuerdo con las características topográficas y geológicas y los datos climatológicos conocidos y disponibles hasta entonces. En años posteriores, se empezarían a calcular los coeficientes de porosidad y almacenamiento y e volumen de recarga natural de agua de cada acuífero, para conocer su potencial real de aprovecha miento.

Un resumen de los diferentes órdenes de magnitud de cada una de las estimaciones descritas se muestra en el cuadro 1. Se aprecia cómo la cantidad de agua calculada fue descendiendo conforme el año del estudio era más reciente. Ello tiene su explicación en el empleo de métodos de estimación más modernos y precisos y en la observación directa en el terreno de los impactos de la extracción. Así, las altas expectativas iniciales sobre los volúmenes de infiltra ción de agua al subsuelo y su potencial real de aprovechamiento fueron disminuyendo con el transcurso del tiempo. Posteriormente, en 1975 y con datos que cubrían el 37\% del territorio nacional, se calculó entre 5 y 10 mil millones de metros cúbicos el volumen

\section{Cuadro 1}

Estimaciones de volúmenes de agua

que se infiltran al subsuelo en México, 1947-1958

\begin{tabular}{|l|c|c|c|}
\hline Autor & Año & $\begin{array}{c}\text { Volumen tota } \\
\left(\mathrm{Mm}^{3}\right)\end{array}$ & $\begin{array}{c}\text { Volumen aprovechable } \\
\left(\mathrm{Mm}^{3}\right)\end{array}$ \\
\hline Adolfo Orive Alba & 1947 & 263,000 & \\
Andrés García Quintero & 1949 & 350,000 & $211,000(1958)$ \\
Alfonso De la O Carreño & $1951-1954$ & 270,000 & \\
Luis Echeagaray & 1958 & 152,000 & 64,000 \\
JorgeTamayo & 1958 & 185,000 & 27,000 \\
\hline
\end{tabular}

Fuente: Elaboración del autor con base en bibliografía anexa. 
renovable de agua subterránea, que se podía extraer de manera permanente. El volumen no renovable almacenado se estimó entre 60 y 80 mil millones de metros cúbicos ( SRH, 1975).

Para Arreguín (1998: 25-26), estos años formaron parte de la segunda etapa en la historia de la geohidrología que denominó "los detonantes del desarrollo de la geohidrología" y que abarcó el periodo de los años 1935-1966. El año de inicio propuesto tuvo como fundamento dos razones: a) ser la fecha en que CharlesV.Theis, considerado el fundador de la moderna hidráulica de pozos, da a conocer su histórica ecuación de flujo no establecido hacia un pozo; y b) porque "más o menos" coincide con los primeros desarrollos masivos de pozos para abastecer de agua a la ciudad de México y a los grandes proyectos de irrigación agrícola de la Costa de Hermosillo, Sonora y el Valle de Santo Domingo, Baja California Sur.

En esta etapa, los problemas y las actividades geohidrológicas giraron en torno al desarrollo y aplicación de nuevas tecnologías en el ámbito de la hidrología subterránea cuantitativa. En ello fue decisivo el reconocimiento de que el agua subterránea "constituye un recurso natural limitado, ante los ejemplos de sobreexplotación de acuíferos que ya empezaban a manifestar efectos perjudiciales e indeseables desde cualquier punto de vista". Estos ejemplos "fueron reto y acicate" para que los técnicos abordaran "con mayor seriedad y empeño los aspectos cuantitativos enfocados a determinar el potencial de los almacenamientos del subsuelo". A los trabajos de prospección indispensables para identificar la presencia de agua subterránea, se agregaron: la determinación de las características hidrodinámicas, el conocimiento de la calidad del agua por alumbrar, la elaboración de un modelo conceptual del comportamiento del acuífero y su modelación.

Dos aspectos notables para el desarrollo de la geohidrología en estos años, continuando con Arreguín (1998: $41-42$ y 49), fueron el desarrollo mismo de los distritos de riego por bombeo y los avances de la industria petrolera en materia de perforación. En los primeros, al principio, la búsqueda de agua subterránea estuvo regida por la "rabdomancia y los zahoríes", pero a medida que adquirió importancia el número de pozos y el área de riego que servían, los usuarios se organizaban, se decretaba la creación del distrito de riego 
respectivo, se tecnificaban la localización y diseño de pozos, el riego, los cultivos y en general la actividad agrícola. No obstante lo anterior, dicho autor reconocía inmediatamente después la falta de control y planeación en su explotación y los impactos negativos que generó:

La perforación de pozos fue anárquica, siempre en el predio del interesado y fuera de consideraciones geohidrológicas regionales. Al paso de los años se fueron notando los efectos de interferencias durante la operación conjunta de los pozos, el abatimiento generalizado de los niveles de agua por sobreexplotaciones locales primeramente, y regionales después, con sus variados efectos secundarios colaterales (I bidem:49).

En cuanto a la tecnología petrolera, fueron cruciales todos los avances en materia de equipos de perforación con sistema rotatorio que se dieron entre 1890 y 1950 y que fueron aplicados en la búsqueda de agua del subsuelo. Entre ellos estuvieron: los nuevos diseños de barrenas, la calidad del acero de las tuberías, el tamaño de las bombas de lodos, los lodos de perforación y los motores de combustión interna, tanto de gas como de diesel, que desplazaron a los de vapor. Estas mejoras se tradujeron en un aumento de la profundidad de perforación, que pasó de los $600 \mathrm{~m}$ en 1900 a casi $7,500 \mathrm{~m}$ en la actualidad, y de la velocidad de penetración, que pasó de unos cuantos metros hasta $100 \mathrm{~m}$ en 24 horas. $^{5}$

Lo relevante en esta etapa y en particular en los distritos de riego por bombeo fue que, a pesar de la falta de certeza sobre la cantidad de agua que almacenaban los acuíferos y, por ende, sobre la cantidad que podía ser explotada sin afectarlos, la extracción del líquido (denominado "libre alumbramiento" o "bombeo libre") comenzó en gran escala sin esperar a que su volumen fuera siquiera preliminarmente cuantificado. Las primeras estimaciones confiables llegarían varios años después, en la década de los años 60 , cuando los abatimientos eran ya significativos en ciertas regiones como la Costa de

\footnotetext{
5 Entre la década de los años 40 y 50 se desarrolló la fabricación de máquinas lla madas "hechizas", copiando técnicas extranjeras, principalmente de la Bucyrus Erie.
} 
Hermosillo y las soluciones posibles parecían llegar demasiado tarde.

\section{Los primeros cálculos de agua aprovechable} en la Costa de Hermosillo

La primera estimación sobre el volumen de agua subterránea que se podía extraer del acuífero de la Costa de Hermosillo sin afectarlo, se le debe al ya citado De la 0 Carreño (1960). Éste señaló una recarga natural del acuífero de $324 \mathrm{Mm}^{3}$ al año, cifra que sería ligeramente inferior a la que calcularía años después el estudio de Ariel Construcciones (1968) de $350 \mathrm{Mm}^{3}$ y que es la más aceptada en la actualidad. Dicho volumen se estimó empleando la metodología de coeficientes de infiltración, la consulta de datos de observación y el auxilio de diversas cartas del comportamiento y naturaleza de las aguas subterráneas como salinidad y abatimiento, que comenzaban a elaborarse ya en el distrito de riego. Incluyó reconocimientos aéreos en avioneta por la zona, rápidos reconocimientos por tierra y obtención de líneas de fotografías aéreas. Su objetivo era determinar si se estaba sobreexplotando el acuífero y recomendar medidas para su solución.

Concluyó que había una extracción de $800 \mathrm{Mm}^{3}$, cifra más de dos veces superior a la alimentación. Alertó sobre "el peligro de la invasión y contaminación con las aguas saladas costeras" de continuar la sobreexplotación, y la probabilidad de que en 7 años más se estuviera bombeando agua salada en la franja más cercana a la costa, cosa que sucedió y se reconoció oficialmente aproximadamente 10 años después. Recomendaba,entre otras cosas, efectuar un estudio geohidrológico preliminar más a detalle, así como un estudio geológico de la región. Ya desde entonces se mencionaba que la alimentación principal del acuífero de la Costa de Hermosillo era la proveniente del arroyo Bacoachi (figura 1). Proponía reducir la extracción a la de 1951 que fue de $387 \mathrm{Mm}^{3}$, así como revestir cana les y nivelar tierras para disminuir el consumo de agua. 
Figura 1

\section{Distrito de riego. Costa de Hermosillo}

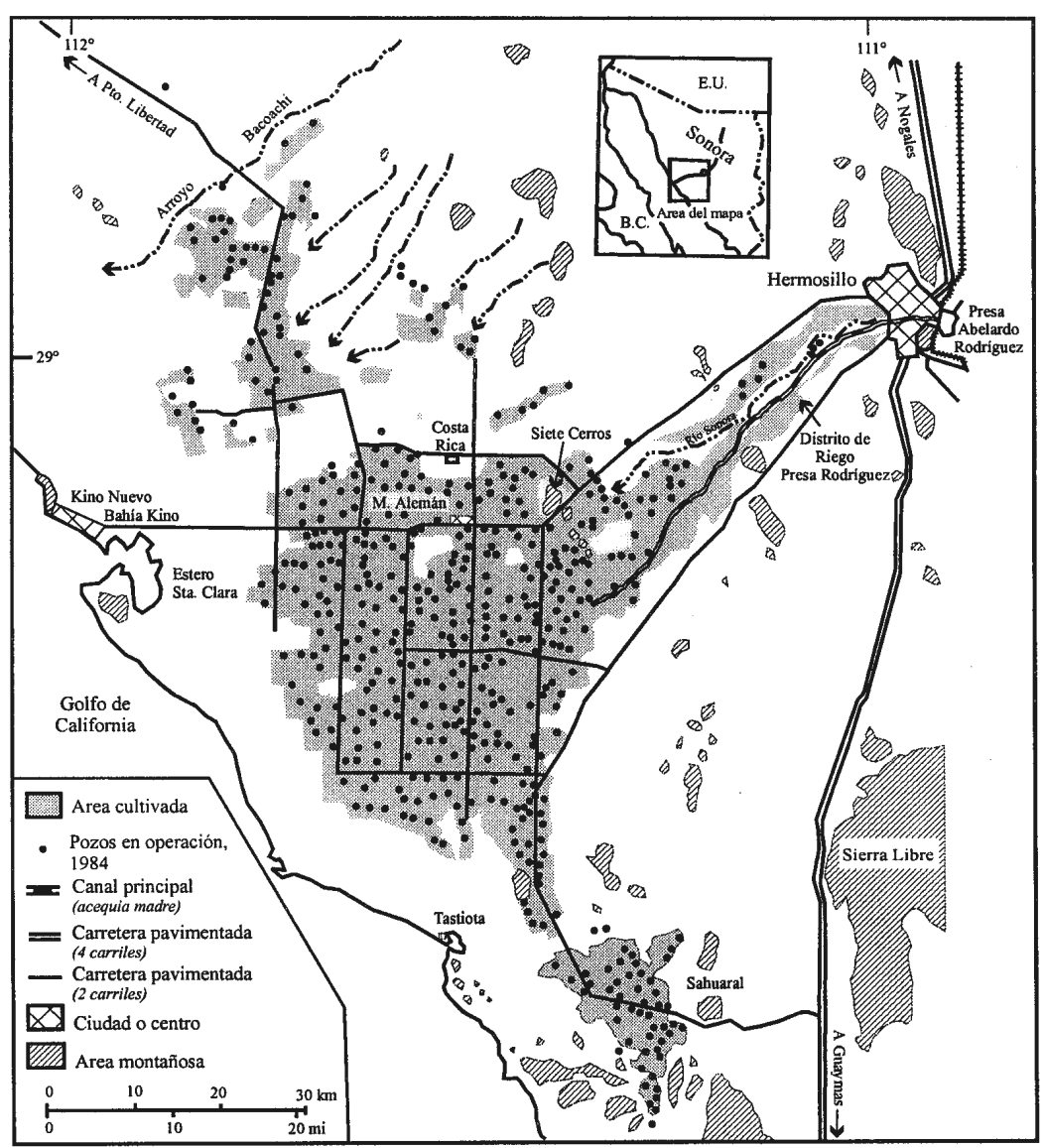

Fuente: West, 1993. 
Específicamente propuso llevar a cabo 200 sondeos eléctricos a una profundidad mínima de $200 \mathrm{~m}$, perforar 10 pozos centinelas y 10 piezométricos para el control de la probable invasión salina y de las presiones, con una profundidad mínima de $\mathbf{1 5 0} \mathrm{m}$. Pugnaba porque las perforaciones fueran de preferencia contratadas con empresas que "garantizaran el éxito de las mismas". Además, recomendaba un estudio gravimétrico para indagar posteriormente si había barreras estructurales en el subsuelo o depresiones que puedan "defender de la intrusión salada" a la Costa de Hermosillo. Este estudio ayudaría a conocer la profundidad y dimensiones de la cuenca subterránea en explotación. ${ }^{6}$

En 1965, apareció publicado el trabajo del ingeniero Angel Jiménez Villalobos Utilizando el método de Hill, basado en el volumen extraído y la variación del nivel estático en un largo periodo de observación, estimó en $570 \mathrm{Mm}^{3}$ el volumen que se podía extraer del subsuelo sin que "se registren abatimientos ni recuperaciones en el nivel de las aguas subterráneas" (Jiménez, 1965:65-81). Su problema fue, según Arreguín (1998: 63), que el método no es aplicable, ya que la cifra mencionada incluía el agua de intrusión marina, es decir, un componente de recarga al acuífero "indeseable por contaminante" y que debía de restarse de las aportaciones tota les determinadas de esta manera.

En este trabajo llaman la atención sus frases: la "posibilidad teórica" de una invasión de las aguas del mar hacia los acuíferos en explotación, la ausencia de este fenómeno en el ciclo agrícola 1962-63 según "la composición química de las aguas extraídas", y la "muy remota posibilidad de avance de las aguas marinas"debido a la exis tencia de cordilleras paralelas a la costa que interrumpen la continuidad de los acuíferos en explotación hacia el mar, salvo la zona del estero Santa Cruz ( cerca de Bahía Kino) constituida por forma ciones muy permeables. Frases que, parecían revelar en cierta medida, una resistencia a aceptar el riesgo de intrusión salina de la que

\footnotetext{
6 Otro estudio relevante realizado por este autor fue llevado a cabo en la región agrícola de Santo Domingo, B. C. S., en donde debido a los volúmenes de extracción y la infiltración de agua al subsuelo calculados, recomendaba "no perforar nuevos pozos" y "no poner en explotación los existentes no equipados". De la 0 Carreño (1961).
} 
hablaban y estaban casi seguros los geólogos si continuaban las altas extracciones del acuífero. Esta diferencia de opinión sería una constante en los estudios realizados durante la década de los años 60 por parte de geólogos e ingenieros agrónomos (Moreno, 2000).

En ese mismo año de 1965, el citado Tamayo olvidó algo de su prudencia expresada años antes en cuanto a la cantidad de agua subterránea aprovechable del subsuelo. En una reunión efectuada en Hermosillo, indicó una recarga total de los acuíferos de la planicie costera de Sonora de $2,000 \mathrm{Mm}^{3}$, siendo el de la Costa de HermosiIlo (al que llamaba también "del río Sonora") el de mayor volumen con $780 \mathrm{Mm}^{3}$, cifra que representaba más del doble de lo estimado por De la 0 Carreño. Señalaba que las cifras "no son muy lejanas a la realidad", y que en el caso de la Costa "tan puede ser buena la cifra mía como la de la secretaría", ya que ésta es de alrededor de 900 $\mathrm{Mm}^{3}$. Lo importante para él era "que es inferior a los 1,000 $\mathrm{Mm}^{3}$, que actualmente se están sacando con las bombas" y "que podrían regarse 230 mil hectáreas", aprovechando este volumen en su tota lidad para riego. Además, observó que en la Costa de Hermosillo $693 \mathrm{Mm}^{3}$ de líquido eran "aguas fósiles", es decir, el $88 \%$ del total (Tamayo, 1965: 265-266).

Conceptualizó con ese nombre a los mantos subterráneos que "no reciben realimentación reciente y que no son sino vestigios de un almacenamiento realizado en épocas geológicas anteriores". Consideraba que estas aguas "no son depósitos regenerables", que puestas bajo explotación "tendrían que agotarse en un plazo más 0 menos largo", y que había que darles un tratamiento de "recurso natural no renovable" El otro tipo de mantos subterráneos existentes eran aquellos que "reciben aportaciones contemporáneas y que, por lo tanto, son reabastecidos" (Tamayo, 1964: 74-75).

Sin embargo, paraTamayo tal presencia de aguasfósiles en la Costa no era problema. El problema era no evaluar con precisión la cantidad de agua almacenada en estos depósitos para prever su agotamiento a una extracción determinada:

...no nos debemos de espantar de que los depósitos sean de aguas fósiles y los vayamos a agotar. Yo creo que los depósitos de 
aguas fósiles es peligroso explotarlos sin saber su volumen, pero en $\mathbf{E}$ momento que sepamos su volumen podemos darle $\mathbf{e}$ mismo tratamiento que a un depósito de mineral, un recurso natural no renovable (1965: 266).

Con esta argumentación parecía justificarse el tipo de agricultura que había criticado, varios años antes en su clásico texto sobre los distritos de riego del noroeste, el reconocido Jacques Chonchol (1957), asesor de la FAO. Al estudiarla y observar sus impactos en el terreno, la definió como una agricultura tipo minería que trata de sa car el mayor provecho a los recursos agua y suelo en poco tiempo sin preocuparse del futuro.

Parte del optimismo de Tamayo se basaba también en el convencimiento de que "dentro de 30 o 40 años" se podría disponer de agua de Alaska en la frontera de México o de los sobrantes del río Santiago en Nayarit mediante una sucesión de obras (conocidas como Plan Hidráulico del Noroeste). Por tal motivo - finalizaba"no nos deberíamos espantar de agotar el agua fósil", si hay la seguridad de que en ese lapso llegará a Sonora "un repuesto de agua pa ra sustituirla" (Tamayo, 1964:267).

Un año después fue publicado en una revista especializada de los Estados Unidos el trabajo de Busch, Matlock y Fogel, (Busch, et al, 1966), quienes un año antes habían realizado un estudio por encargo del organismo que representa a los agricultores privados más importantes de la región: la Asociación de Organismos Agrícolas del Norte de Sonora (Busch, $\notin$ al, 1965). Se citan ambos documentos, escritos por los mismos autores y sobre la misma región objeto de estudio, ya que existen pequeñas diferencias entre las preguntas y el contenido de cada uno de ellos. Por su interés, se cita en mayor medida el contenido del documento entregado a los agricultores como consultores, y no el publicado como profesores de ingeniería agrícola de la Universidad de Arizona en Tucson los dos primeros, y como hidrólogo de la misma institución el tercero.

Las preguntas que planteó el estudio fueron dos: ¿Cuál es la cantidad de agua subterránea existente y la cantidad bombeable sin la intrusión de agua salada durante un largo periodo de tiempo? y ¿pueden mejorarse los métodos de riego para ahorrar agua? Entre 
las técnicas empleadas estuvieron: evaluaciones de las técnicas de riego en el campo, análisis de la tierra, análisis de la transmisibilidad del acuífero de acuerdo con el método propuesto por el U.S. Geological Survey (presentado en el documento "Suministro de agua 1464") y tasas de introducción de agua salada. También se estimaron los "recursos de agua potencial", mediante el análisis de la información de las pruebas de bombeo de 20 pozos.

Concluyó que la aportación de agua dulce es menor a la mitad del agua bombeada y que el agua almacenada representaba una posible explotación de 100 años, siempre y cuando se minimizaran los efectos de la intrusión salina y del exceso de bombeo. Si el agua bombeada en los ciclos agrícolas 1963-1964 y 1964-1965 fue superior a los $1,000 \mathrm{Mm}^{3}$ cada año, esto quiere decir que calcularon una aportación natural al acuífero menor a los $500 \mathrm{Mm}^{3}$. La cantidad de agua disponible se estimó en la asombrosa cifra de 100,000 $\mathrm{Mm}^{3}$ de agua, la cual ocupaba una tercera parte del volumen total del acuífero.

Los niveles del agua en la mayor parte del distrito se encontra ban bajo el nivel del mar y el abatimiento anual era de 1 a $1.5 \mathrm{~m}$. El nivel del agua bombeada estaba a una profundidad promedio de $70 \mathrm{~m}$ y se calculaba que en 20 años estaría a $100 \mathrm{~m}$. La velocidad con que el agua salada entraba a los campos o "usurpaba el área de cultivo" se estimó en 21 metros al año. Señaló las "oportunidades limitadas" para aumentar la recarga acuífera con agua proveniente de las precipitaciones, ya que el valor de la evaporación era 10 veces mayor. Concluyó que el agua no era utilizada eficientemente según los métodos de riego observados y que el riego por aspersión podría ahorrar hasta $50 \%$ del volumen de agua empleada por otros métodos. Por último, apuntó que un descenso en la sobreexplotación del agua podía realizarse mediante la reducción de la superficie sembrada, un cambio en los modelos de siembra, la importación de agua de otras cuencas o la captura de un mayor porcentaje de la precipitación.

Cabe resaltar que el agua bombeada en los dos ciclos agrícolas mencionados representó el volumen máximo de extracción en la historia de la Costa, y significó casi 4 veces más que la recarga na 
tural calculada por De la 0 Carreño. Este volumen de agua fue empleado para regar una superficie de 126 y 130 mil hectáreas en ambos ciclos, mediante la operación de un total de 493 pozos.

Un poco más adelante se presentaron los resultados del estudio elaborado por una empresa israelí a solicitud de la \$RH (Tahal, 1966). Si bien éste no incluyó ninguna estimación del agua almacenada en el acuífero ni de su recarga natural, se cita aquí porque recomendó incrementar los métodos de recolección de datos y evaluarlos correctamente para la mejor administración de los recursos de agua en el distrito. Para subsanar la "magra información existente", recomendaba perforar 50 pozos de investigación y observación y correlacionar sus resultados con mediciones geofísicas o geoeléctricas, así como el uso extensivo de computadores análogos o modelos hidrológicos. En general, hablaba de un "manejo científico de las aguas subterráneas". Por último, propuso un plan preliminar para la reubicación de pozos y estudios de previabilidad de proyectos para transportar agua de otras cuencas a la Costa de Hermosillo y proyectos de desalinización de agua de mar.

Dos años después concluyó el trabajo que constituye el estudio más detallado realizado sobre el acuífero de la Costa de HermosiIlo hasta la fecha (Ariel,1968). Su elaboración fue el resultado de un estudio preliminar efectuado en 1967 que se consideró como directo de tercer orden. ${ }^{7}$ En este primer estudio se cuantificó "una magnitud probable" de la recarga media del acuífero de $350 \mathrm{Mm}^{3}$, mediante la aplicación de una "teoría para abatimientos medios re-

\footnotetext{
7 Según la terminología geológica, eran estudios de tercer orden aquellos cuyas observaciones y conclusiones no podían considerarse definitivas. Se apoyaban en el uso de información directa (de los acuíferos mismos e incluía estratigrafía, piezometría, caudales de entrada y salida, y resultados de pruebas de bombeo) e información indirecta (mediciones y observaciones en superficie como levantamiento hidrogeológico, regis tros hidrológicos y climáticos, estimaciones de evapotranspiración,etcétera). Para ser de segundo orden, la información anterior tenía que complementarse con la aplicación de ciertas leyes e hipótesis deducidas de la observación del abatimiento, para estimar directamente los caudales que fluyen por los acuíferos. Para ser de primer orden tenía que crear un modelo geohidrológico matemático. Lo ideal sería un estudio hidrológico integral, es decir, aquel que manejara conjuntamente el sistema hidrológico superficial y el sistema hidrológico subterráneo, estableciendo sus mutuas relaciones. Larios (1968: 17-20).
} 
gionales" y utilizando la historia conocida de los abatimientos y las extracciones en el distrito. Lo que hizo el segundo estudio fue confirmar la cifra anterior, la cual todavía hoy es la cifra oficial manejada por las autoridades respectivas. Otro de sus objetivos fue "investigar la posible existencia de nuevas fuentes de agua subterránea en la región, que ayuden a cubrir el déficit existente".

Ambos estudios incluyeron la perforación de 28 pozos por parte de la compañía privada Perforadora Latina y una prospección geoeléctrica realizada por Geofimex,S.A. El propósito era "explorar más allá de los límites de la arcilla azul, frontera situada alrededor de los $\mathbf{1 5 0} \mathrm{m}$ de profundidad y que nunca antes se había traspasa do". En total, 11 fueron pozos de bombeo, 14 de observación pa ra prueba de bombeo y lecturas piezométricas periódicas y 3 para observar el avance de la intrusión salina. Aunque su profundidad promedio fue de 150 a $200 \mathrm{~m}$, algunos llegaron a tener más de 700 metros. La profundidad máxima alcanzada en la prospección geoeléctrica fue de 1,500 metros.

Según su representante, el ingeniero José Arreguín, este estudio que implicó una profundidad de investigación a más de $1,000 \mathrm{~m}$ de profundidad, algo insólito en aquellos años en el medio, vino a "salvar el prestigio de la geofísica". Esto se debía a los pobres resultados obtenidos hasta entonces por medio de dichos métodos de prospección y que se encontraban desprestigiados por el grupo de técnicos que ejercían la especialidad, salvo honrosas excepciones (Arreguín, 1998: 70-71).

Este trabajo fue el que determinó también las caracteństicas bá sicas y el funcionamiento del acuífero conocidas hasta la actualidad: la existencia de dos acuíferos interconectados entre sí, separados por un manto arcilloso de origen marino con espesor variable. El primer acuífero (llamado superior) con un espesor de $200 \mathrm{~m}$; el manto arcilloso con espesor de $\mathbf{4 0 0} \mathrm{m}$ cerca del mar y que desaparece al interior del continente; y el segundo acuífero (llamado inferior) con espesores variables hasta los $700 \mathrm{~m}$ de profundidad, lugar donde se presenta el basamento granítico regional. La alimentación proviene en $\mathbf{2 2} \%$ de las partes altas de los ríos Bacoachi y Sonora y el $\mathbf{7 8 \%}$ restante del acuífero inferior a través del manto arcilloso, ignorándose la alimentación total de este último (figura 2). 


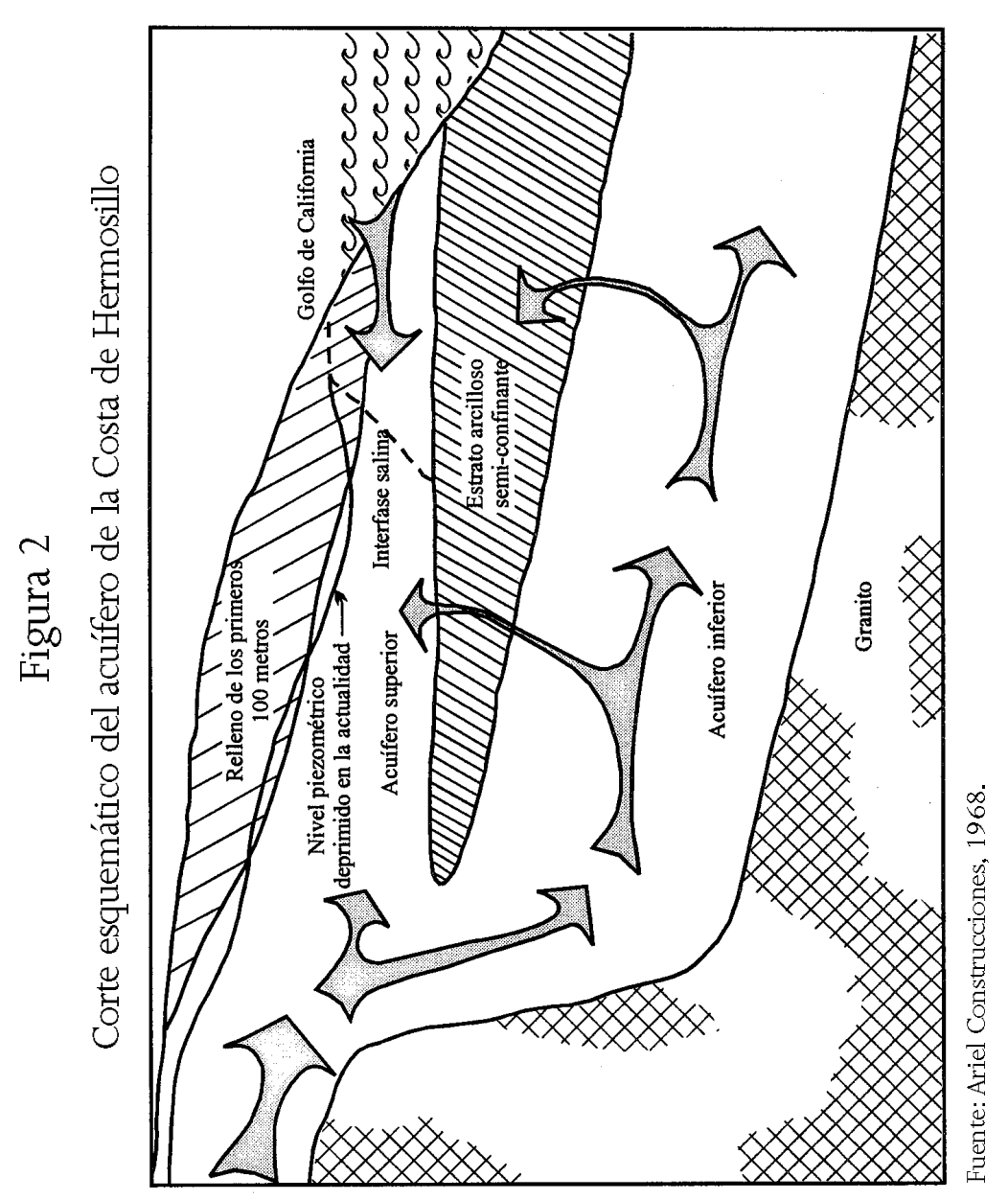


Un aspecto fundamental de este estudio fue el descubrimiento del segundo acuífero, que hasta entonces se desconocía. Sobra decir lo que este descubrimiento significó en momentos en que los agricultores sufrían numerosos problemas derivados de la aplicación del reglamento de explotación que había reducido en $24 \%$ las extracciones de agua en el periodo de los años 1964-1968. Fue tal la euforia que generó, que el entonces secretario de Recursos Hidráulicos José Hernández Terán declaró en una reunión efectuada en Hermosillo que el descubrimiento de este manto junto con otros que se localizaron casi el mismo tiempo en Guaymas y Caborca, "hacen prever que la veda para la perforación de pozos en vastas zonas del territorio sonorense sea levantada" (El Imparcial, 1968).

Incluso ese mismo día, el funcionario federal se reunió con representantes de agricultores para solicitarles su apoyo financiero para acelerar los trabajos y concluirlos. Esta colaboración podría provenir de una cuota adicional en las extracciones de agua de ese entonces, 0 bien con perforaciones que los propios agricultores financien en la "inteligencia de que dichos pozos se utilizarán en provecho propio". Cuatro meses después, el citado diario daba cuenta de la aportación de 2 millones de pesos - de un total de 4 millones- por parte de los agricultores de la Costa a la dependencia federal para $\mathbf{e}$ incremento de las investigaciones de aguas subterrá neas en la región (El Impardal, 1968).

E descubrimiento de este nuevo acuífero y la elaboración del estudio citado se dieron en el contexto de la realización de numerosos estudios geohidrológicos en el país promovidos por la Dirección de Aguas Subterráneas de la \$R, bajo la dirección del ingeniero Ignacio Sáinz Ortiz. Creada en 1966, entre las atribuciones de esta dependencia estaban "evaluar los recursos de aguas subterráneas" en diversas regiones del territorio, así como vigilar que su explotación "se efectúe racional y eficientemente". De acuerdo con Arreguín, este momento marcó el inicio de la tercera etapa en la historia de la geohidrología definida como "consolidación y difusión de la hidrología subterránea (1966-1995)". Los estudios se llevaron a cabo en áreas consideradas como críticas o con problemas graves. Entre otras regiones en donde hubo resultados positivos en la perforación de pozos para obtener agua estuvieron: las ciudades de Monterrey y 
León, los valles del Mezquital, Mexicali, Mesa de San Luis y Ciudad Juárez, y el desierto del Vizcaíno.8

En forma similar a las expectativas que hubo en cuanto a la disponibilidad de agua subterránea en el país, el potencial de la recarga acuífera en la Costa de Hermosillo fue disminuyendo conforme el paso del tiempo. La única excepción lo fue el primer estudio rea lizado por De la 0 Carreño en 1960, quien desde el principio estimó la cifra más baja de todas las propuestas (cuadro 2).

\section{Cuadro 2}

Estimaciones de volúmenes de recarga acuífera en la Costa de Hermosillo, 1960-1968

\begin{tabular}{|l|c|c|}
\hline Autor & Año & Volumen tota $\left(\mathrm{Mm}^{3}\right)$ \\
\hline Alfonso De la O Carreño & 1960 & 324 \\
Ángel Jiménez Villalobos & 1965 & 570 \\
Jorge Tamayo & 1965 & 780 \\
Busch,Matlock y Foguel & 1966 & -500 \\
Ariel Construcciones & 1968 & 350 \\
\hline
\end{tabular}

Fuente: Elaboración del autor con base en bibliografía anexa

Así, la cifra de recarga natural en el acuífero de la Costa de Hermosillo de $350 \mathrm{Mm}^{3}$ llegó 23 años después de la perforación del primer pozo profundo en la región. $Y$ varios años después de la emisión de diversos ordenamientos jurídicos tendientes a regular la extracción de agua, tales como: 4 decretos de veda (1951,1954, 1963 y 1967), 9 el citado reglamento de explotación (1963) que en los hechos constituyó el primer programa de reducción de extrac-

\footnotetext{
8 La citada empresa Ariel tuvo a su cargo también la realización de estudios en los valles de Mexicali, Baja California;Guaymas,Sonora, y Santo Domingo, Baja California Sur. Arreguín (1998: 67-73).

9 Formalmente el decreto de 1951 es el único con el nombre "decreto de veda"; los 3 restantes son "ampliaciones" del área de veda original.
} 
ciones, un reglamento de operación del distrito de riego (1966) y la creación de un consejo de conservación de los recursos geohidrológicos (1956).

De esta forma, el conocimiento de su volumen parecía llegar demasiado tarde, cuando el punto crítico de bombeo del acuífero se encontraba a cas $30 \mathrm{~m}$ de profundidad y la intrusión salina era ya casi un hecho.Y lo que es peor, con el hallazgo paralelo de que había todavía un segundo rico manto de agua aún sin explotar. Producto en parte de ese descubrimiento, las extracciones de agua volverían a aumentar a un volumen anual entre 800 y $900 \mathrm{Mm}^{3}$ en el periodo 1968-1977, cifra que sería similar a la manifestada en los primeros diez años de vida del distrito (gráfica 1). De hecho, en el ciclo 1969-1970 se alcanzaría el máximo histórico de superficie sembra da: 132 mil hectáreas. Terminado ese periodo de regreso a una alta extracción, dio inicio el segundo programa de reducción de extracciones que concluyó en 1990, el cual, dicho sea de paso, tampoco logró igualar los volúmenes de extracción de agua con la recarga natural. En el presente, la sobreexplotación del acuífero continúa, con un volumen de extracción anual de $460 \mathrm{Mm}^{3}$. El nivel estático está a 60 metros bajo el nivel del mar y la intrusión salina aumentó sus valores de salinidad de 500 hasta 8,000 partes por millón en una franja de 10 a 15 km de ancho (CNA, 1999: 6, 15).

\section{Consideraciones finales}

Este breve recorrido por la forma en que evolucionó el conocimiento del agua subterránea en el país y los resultados principales de los estudios realizados sobre el acuífero de la Costa de Hermosillo hasta finales de los años sesenta muestra, en ambos casos, que el conocimiento sobre sus características y potencial fue a la zaga de su explotación en gran escala Y cuando éstas se alcanzaron a determinar, como en el caso de la región de estudio, en lugar de propiciar la utilización adecuada del acuífero, provocaron una mayor extracción de agua. También muestra que ante los déficits de líquido, hubo mayor interés por encontrar nuevos depósitos, que por fomentar su uso eficiente y conservación. En igual forma, refleja que las estimaciones 


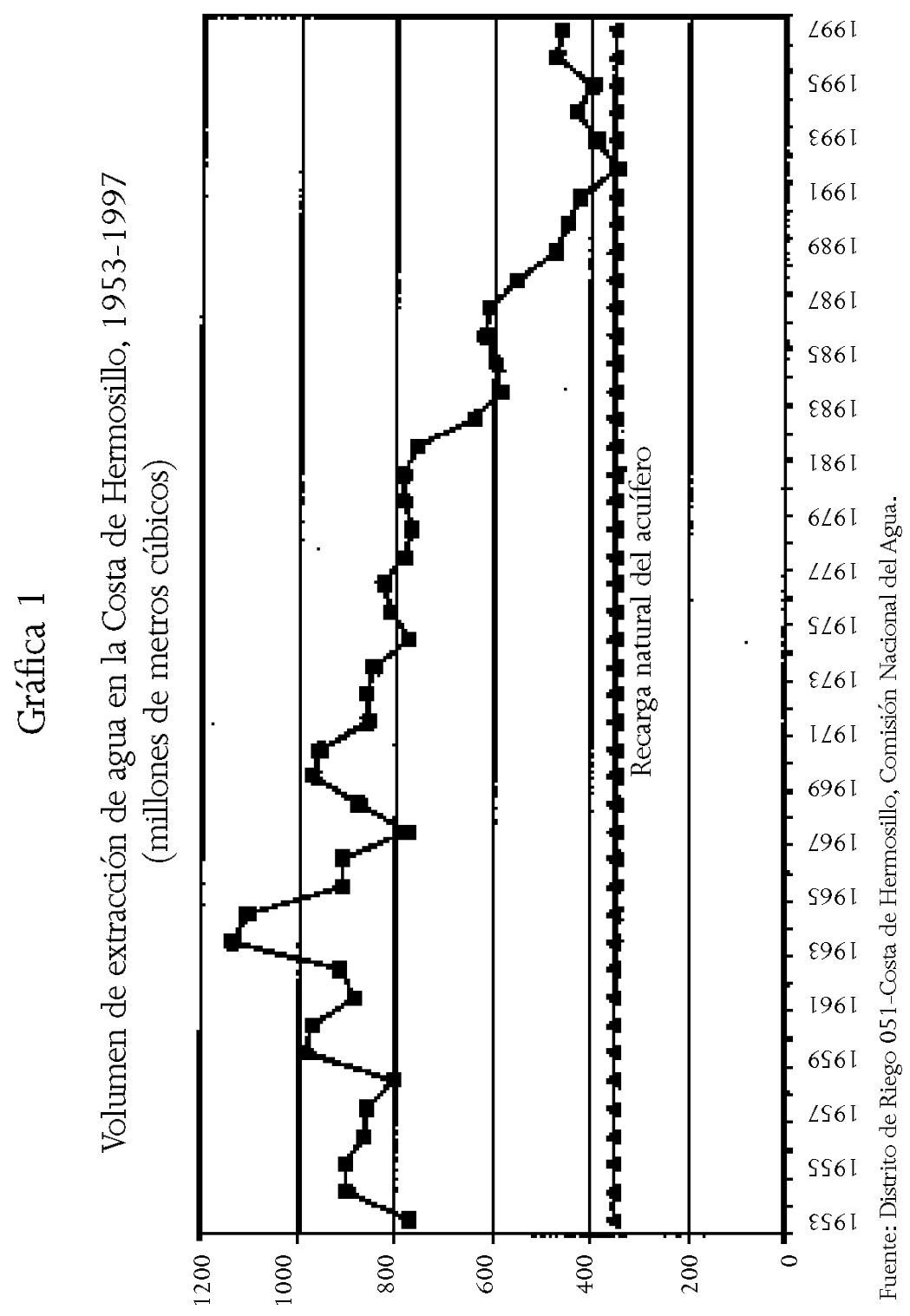


iniciales de la cantidad de agua subterránea infiltrada y aprovecha ble, tanto en el país como en la Costa de Hermosillo, fueron disminuyendo en la medida en que los métodos empleados obtuvieron información directa proveniente de los acuíferos mismos.

Asimismo, que la Costa fue una zona privilegiada en cuanto a elaboración de estudios en relación con otras zonas del país, no sólo por su número y metodología empleadas, sino por el tipo de personas involucradas en ellos. Dos casos fueron los citados De la 0 Carreño y Arreguín, quienes fueron, director de geología de la misma SRH y pionero de muchos estudios geohidrológicos el primero, así como uno de los consultores privados más importantes del pás el segundo. 0 tro caso fue el de los investigadores procedentes de la Universidad de Arizona en Tucson.Además, dicho recorrido reveló que la mayor parte de los ordenamientos jurídicos emitidos en la Costa de Hermosillo para regular la explotación del agua fueron inoperantes.

Desgraciadamente, la preocupación e interés por conocer la cantidad de agua que se puede extraer del acuífero sin afectarlo negativamente desapareció después del estudio de la empresaAriel en 1968. En los años siguientes y hasta la actualidad, no volvería a efectuarse ningún otro con detalle para determinar si el volumen de la recarga natural había variado con el transcurso de los años y poder planear asi su aprovechamiento cabal y sostenible durante un largo periodo de tiempo. La razón de ello puede ser que ni la autoridad competente, ni los productores agrícolas, han estado realmente interesados en conocer cuál es su situación, confiando tal vez en la potencialidad del segundo acuífero descubierto desde entonces. En este sentido, documentos internos de la propia dependencia encargada de su administración han reconocido que "cabe la posibilidad" de que el volumen de la recarga sea menor a $350 \mathrm{Mm}^{3}$ debido al incremento de las extracciones en los cañones de los ríos Sonora, San Miguel Zanjón y Bacoachi (CNA, 1992). Esto, si se demuestra en un estudio, podría tener como consecuencia inmediata la disminución de la dotación de agua actual a los agricultores.

Sobre este tema, lo único que se ha hecho en años recientes es un estudio de balance de agua en la Costa para el ciclo específico 1986-1987 realizado por la geóloga Lourdes Vega de la Universi- 
dad de Sonora Dicho balance se llevó a cabo empleando datos de climatología, planos de elevaciones de niveles estáticos, planos de transmisividades y volúmenes de bombeo. Entre sus conclusiones principales estuvo una estimación de entrada de agua (dulce y sala da) al acuífero de $318 \mathrm{Mm}^{3}$, mientras el bombeo fue de $610 \mathrm{Mm}^{3}$, es decir, una sobreexplotación de 91\% (Vega, 1997: 40).

Así, las preocupaciones principales de los estudios en torno al agua del acuífero en las últimas tres décadas se centraron en temas como: la intrusión salina y el empleo de modelos matemáticos pa ra observar y predecir su comportamiento futuro, modelos óptimos de patrón de cultivos, análisis económicos de los usos del agua y necesidades técnicas de información ante su escasez. Otros temas abordados fueron: la gestión ambiental a nivel de cuenca hidrográfica, percepción sobre la transferencia del distrito de riego a los usuarios, sondeos geofísicos para investigar su zona vadosa, y oportunidades y barreras relacionadas con el desarrollo sostenible (Moreno, 2000).

El tema de la recarga natural de agua al acuífero no fue objeto de estudio ya de ningún tesista, centro de investigación, dependencia gubernamental o consultoría nacional o extranjera. Como tampoco sería interés de los propios productores, no obstante ser el grupo más afectado por esta problemática. A nivel gubernamental, lo único que continuaría efectuándose sería la medición del nivel de abatimiento del acuífero mediante el paro del bombeo en todos los pozos del distrito durante casi una semana al año, cuya evaluación es utilizada para calcular la extracción realizada y programar la dotación de agua disponible para los ciclos agrícolas siguientes

Esta ausencia de conocimiento en cuanto a la cantidad de agua aprovechable, así como la falta de un estudio geodrológico completo y actualizado sobre el acuífero de la Costa de Hermosillo, adquiere hoy día una relevancia mayor ante el planteamiento de nuevos proyectos de aprovechamiento de agua en la región. Tal es el caso de la construcción de una planta desaladora para resolver los problemas de abastecimiento de líquido de la ciudad de Hermosillo. Este proyecto contempla, entre otros aspectos, perforar una batería de pozos profundos localizados en el "área salobre" del subsuelo cercana al litoral para extraer 3, 400 litros por segundo y desalarla por el proceso de ósmosis inversa. El objetivo es producir $2,500 \mathrm{l} / \mathrm{seg}$ de agua 
potable (216, $000 \mathrm{mil} \mathrm{m}^{3}$ / día), conducirla a la ciudad a través de un acueducto de $110 \mathrm{~km}$, e invertir la línea interfase que divide los mantos de agua dulce y agua salada formando una mayor barrera entre ambas (CNA, 1999:12-14).

Todo esto se plantea sin tener el conocimiento científico actual sobre la dinámica y comportamiento del acuífero después de $\mathbf{5 0}$ años de explotación intensiva, y sin el cual resultará difícil predecir y prevenir los impactos negativos derivados de la construcción de este tipo de obras con un alto componente de innovación tecnológica, tal y como - según hemos visto- ocurrió en el pasado. Entre los inconvenientes de estas obras están: su requerimiento de una alta inversión en recursos financieros para llevarlas a cabo y que las experiencias exitosas en México están documentadas únicamente pa ra plantas de uso industrial y uso doméstico en localidades pequeñas, cuya capacidad individual no rebasa los $3,000 \mathrm{~m}^{3}$ / día. 0 sea, una capacidad casi 100 veces menor a la de la planta que se piensa construir. Además, su construcción y operación genera una gran cantidad de impactos ambientales (Arreguín y Martín, 2000).

Ante ello no es casual que, debido a la falta de conocimiento y difusión pública de la información científica y económica que da sustento a este proyecto de planta desaladora, investigadores de la región cuestionen el alto costo para el contribuyente de la misma en relación con otras opciones, como lo puede ser la compra de derechos de agua subterránea a los propios agricultores (Búrquez y Martínez-Yrízar, 2000:281-283).

Por estas razones, en los próximos meses resultará de la mayor prioridad subsanar este vacío de información mediante la realiza ción de un nuevo estudio geohidrológico del acuífero de la Costa antes del inicio de la obra mencionada. Este estudio podría llevarse a cabo con la participación de los diversos centros de investigación existentes en la entidad y sus recursos humanos especializados en el tema, en colaboración con las dependencias encargadas de su ges tión. Su financiamiento podría correr a cargo de los sectores involucrados en el proyecto como los gobiernos federal, estatal y municipal, las organizaciones de productores agrícolas, y los organismos financieros internacionales como el Banco Mundial. Con ello se evi- 
taría la presencia, de nueva cuenta, del desfase entre conocimiento y explotación de agua que se manifestó en décadas pasadas.

Para evitar lo que sucedió cuando se comprobó la existencia de un mayor potencial del acuífero a finales de los años 60, dicho estudio debería ser la "piedra de toque" o el insumo de información básica, a partir del cual se promoviera el debate público, abierto y transparente entre los sectores directamente interesados y la comunidad en general, acerca de cuáles son sus ventajas y desventajas, si es o no una opción viable para la ciudad o la región agnícola y si hay otras posi bilidades por explorar. Igualmente podría servir para eva luar si cuenta con el respaldo de la población de la ciudad o de los mismos productores del campo quienes son los actuales concesionarios del recurso. Con esta participación colectiva y consensada podrían evitarse conflictos sociales que obstaculicen el desarrollo y funcionamiento posterior de la obra y pongan en riesgo la inversión realizada en ella.

De no hacer dicho estudio, o hacerlo después de iniciada la obra sólo para cumplir un trámite administrativo más de una decisión gubernamental tomada a priori, posiblemente en el corto plazo se manifiesten sus altos costos ecológicos sobre la naturaleza y el acuífero de la Costa, sus costos sociales sobre los productores y trabajadores agrícolas y sus costos económicos sobre toda la pobla ción de la ciudad de Hermosillo. Se repetiría aś, una vez más, otro negro capítulo en la historia de la explotación sin conocimiento, sin planeación y sin control de uno de los recursos naturales más preciados del desierto: el agua subterránea.

Recibido en diciembre de 1999 Revisado en marzo del 2000

\section{Bibliografía}

Aboites, Luis (1998), "Notas sobre el optimismo mexicano y los vínculos entre geografía, ingeniería hidráulica y política (19261976)", en Agua, medio ambiente y desarrollo en M éxico, Memorias del xx Coloquio de Antropología e Historia Regionales, El Colegio de Michoacán, pp. 158-167. 
Alanís Patiño, Emilio (1950), "Las tierras de riego", Problemas agríco las e industriales de M éxico, vol. II, no. 2, abril-junio.

Alcalá, Maximino (1927), "Hidrología subterránea de las cuencas de los ríos Magdalena y Altar en el estado de Sonora", Folleto de di vulgación, no. 25, Instituto Geológico de México.

Ariel Construcciones (1968), Estudio hi drogeológico completo de los acuíferos de la Costa de Hermosillo.

Arreguín, José (1998), Aportes a la historia de la geohidrología en México 1890-1995, ClesAs-Asociación Geohidrológica Mexicana, A.C.

Arreguín, Felipe y Alejandra Martín (2000), "Desalinización del agua", Ingeni ería hidráulica en México, vol. xv, no. 1, enero-abril, pp. 27-49.

Blásquez, Luis (1926), “Hidrología subterránea de las cuencas de los ríos de la Concepción y de Sonoyta, Distrito de Altar, estado de Sonora", Folleto dedivul gación, no. 22, Instituto Geológico de México.

Busch, C. D., W. G. Matlock y M. M. Fogel (1966), "Utilization of Water Resources in a Coastal Groundwater Basin", Journal of Soil andW ater Conservation, septiembre-octubre, pp. 163-169.

Busch, Matlock and Associates (1965), Informe final a la Asociación de Or ganismos Agrícolas del Norte de Sonora.

Búrquez,Alberto y Angelina, Martínez-Irízar (2000), "El desarrollo económico y la conservación de los recursos naturales", en Igna cio Almada Bay (comp.), Sonora 2000 a debate, El Colegio de SonoraCal y Arena, pp. 267-333.

Comisión Nacional del Agua Gerencia Estatal en Sonora (1992), Ca racterísticas de los acuíferos de los valles de Guaymas, Costa de Hermosillo y $\mathrm{Ca}$ borca,Sonora. 
Comisión Nacional del Agua (1999), La prioridad es el agua.

Chonchol, Jacques (1957), Los distritos de riego de noroeste Tenencia y apro vechamiento de la tierra, Centro de Investigaciones Agrarias.

De la 0 Carreño, Alfonso (1951), Las provincias geohidrológicas de México (primera parte), Boletín no. 56, Instituto de Geología, UNAM.

(1954), Las provincias geohidrológicas deM éxico (segunda parte), Boletín no. 56, Instituto de Geología, UNAM.

(1960), Condiciones geológicas y geohidrológicas generales de la Costa de Hermosillo, Sonora, Secretaría de Recursos Hidráulicos.

(1961), "Estudio geohidrológico de la región de Santo Domingo, territorio de Baja California", Ingeniería hidráulica en M é xico, enero-marzo, pp. 57-68.

Dumble, E. T. (1901), "El valle de Guaymas", El Minero M exicano, no. 13, tomo XXXVII, versión española.

El Impardial (1968), "Serán salvación de la Costa los nuevos mantos acuíferos También de Caborca y Guaymas, afírmase", 9 de abril.

(1968), 10 de agosto.

Flores, Teodoro (1929), Reconocimientos geológicos en la región central del es tado de Sonora, Boletín no. 49, Instituto Geológico de México.

Jiménez, Angel (1965), "Condiciones de las aguas subterráneas en el distrito de riego no. 51, Costa de Hermosillo, Sonora", Ingenie ría hidráulica en M éxico, vol. xix, no. 3, pp. 65-81.

Larios, Jesús (1968), Estudios hidrogelógicos en la Costa de Hermosillo, tesis de licenciatura en ingeniería, Universidad de Sonora. 
McGee, William (1980), Los seris, Instituto Nacional Indigenista, edición en español de la obra original publicada en inglés en 1898.

Moreno, José Luis (2000), “Apropiación y sobreexplotación del agua subterránea en la Costa de Hermosillo 1945-2000", versión preliminar, tesis doctoral.

Orive Alba,Adolfo (1946), "La política de irrigación”, Problemas eco nómico-agrícolas de M éxico, no. 2, octubre-diciembre, pp. 101-126.

(1960), La política de irrigación en México, Fondo de Cultura Económica.

Secretaría de Recursos Hidráulicos (1975), Plan N acional Hidráulico.

Tahal Water Planning (1966), Informe de estudio de reconocimiento: Costa de Hermosillo-Distrito de riego no. 51, estado de Sonora, Secretaría de Recursos Hidráulicos.

Tamayo, Jorge (1958), El aprovechamiento del agua y de suelo en M éxico, Secretaría de Recursos Hidráulicos.

(1964), El problema fundamental de la agricultura mexicana, México, Instituto Mexicano de Investigaciones Económicas.

(1965), "Aprovechamiento de recursos hidráulicos subterráneos", Desarrollo integral del estado de Sonora, Gobierno del Estado de Sonora y Comisión Mexicana Pro-Alianza para el Progreso, pp. 262-267.

Vega, Lourdes y Juan Saiz (1997), "Estudio de balance de agua de la zona agrícola de la Costa de Hermosillo", Primer Seminario Acuí feros Costeros de Sonora, Hermosillo, Departamento de Agricultura y Ganadería, Universidad de Sonora, noviembre, pp. 30-45.

West, Robert (1993), Sonora Its Geographical Personality, U niversity of Texas Press, Austin. 
